\title{
Pair Production in Non-Perturbative QCD
}

\author{
Salah Hamieh \\ Department of Physics, Faculty of Sciences, Lebanese University, Beirut, Lebanon \\ Email: hamiehs@yahoo.fr
}

Received 8 January 2014; revised 5 February 2014; accepted 3 March 2014

Copyright (C) 2014 by author and Scientific Research Publishing Inc.

This work is licensed under the Creative Commons Attribution International License (CC BY).

http://creativecommons.org/licenses/by/4.0/

(c) (i) Open Access

\begin{abstract}
In this paper, a method to calculate the vacuum to vacuum transition amplitude in the presence of a non-abelian background field is introduced. The number of non-perturbative quark-antiquark produced per unit time, per unit volume and per unit transverse momentum from a given constant chromo-electric field is calculated and its application to quark-gluon plasma is presented.
\end{abstract}

\section{Keywords}

\section{Pair Production, Non-Perturbative QCD}

\section{Introduction}

Lattice QCD predicts a phase transition from Hadrons gaz (HG) to quark-gluon plasma (QGP) at deconfinement temperature, $\mathrm{T} \sim 170 \mathrm{MeV}$. It is believed that QGP has been produced in relativistic heavy ions collision [1]-[4] where in the initial pre-equilibrium stage of QGP about half the total center-of-mass energy, $E_{c m}$, goes into the production of a semi-classical gluon field [5]-[17]. Therefeore, to study the production of a QGP from a classical chromo field, it is necessary to know how quarks and gluons are formed from the latter. The production rate of quark-antiquark from a given constant chromo-electric field $E^{a}$ has been derived in Ref. [18] and the integrated $p_{T}$ distribution has been obtained in [19]-[22] (for a review see [23]).

In this short technical note, we will extend the results of Ref. [18] to a general constant background field. The method presented here may simplify the complexity found in the Non-perturbative QCD calculations. Also, the obtained $p_{T}$ distribution for quark (antiquark) production can be used in the analysis of the experimental results at the RHIC and the LHC colliders.

The paper is organized as follows: in the next section, we will calculate the one loop effective action needed in the evaluation of the $p_{T}$ distribution of the quark (antiquark) production. In Section 3 the $p_{T}$ distribution is presented. Finally, in Section 4, an application to heavy ion collision is given.

\section{The One Loop Effective Action}

As described in the above section, we will evaluate here the one loop effective action in the presence of a 
constant chromo-field. For this purpose, we start from the QCD Lagrangian density for a quark in a non-abelian background field $A_{\mu}^{a}$ which is given by

$$
\mathcal{L}=\bar{\psi}\left[\left(\not p-g T^{a} A^{a}\right)-m\right] \psi=\bar{\psi} D[A] \psi,
$$

Then the vacuum to vacuum transition amplitude is given by

$$
\langle 0 \mid 0\rangle=\frac{\int \mathcal{D} \bar{\psi} \mathcal{D} \psi \mathrm{e}^{\mathrm{i} \int \mathrm{d}^{4} x \bar{\psi} D[A] \psi}}{\int \mathcal{D} \bar{\psi} \mathcal{D} \psi \mathrm{e}^{\mathrm{i} \int \mathrm{d}^{4} x \bar{\psi} D[0] \psi}}=\operatorname{Det}[D[A]] / \operatorname{Det}[D[0]] .
$$

And the one loop effective action can be written in this form

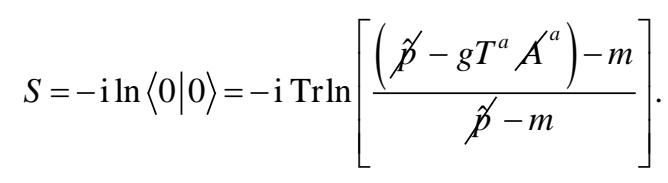

Thus, using the invariance of trace under transposition and the following relation

$$
\ln \frac{a}{b}=\int_{0}^{\infty} \frac{\mathrm{d} s}{s}\left[\mathrm{e}^{\mathrm{is}(b+\mathrm{i} \epsilon)}-\mathrm{e}^{\mathrm{i} s(a+\mathrm{i} \epsilon)}\right]
$$

we obtain the following expression ${ }^{1}$

$$
2 S=\mathrm{i} \operatorname{Tr} \int_{0}^{\infty} \frac{\mathrm{d} s}{s}\left[\operatorname{expis}\left[\left(\hat{p}-g T^{a} A^{a}\right)^{2}+\frac{g}{2} \sigma_{\mu v} T^{a} F^{a \mu \nu}-m^{2}+\mathrm{i} \epsilon\right]-\operatorname{expis}\left[\hat{p}^{2}-m^{2}+\mathrm{i} \epsilon\right]\right] .
$$

The quickest way to calculate the effective action is to work in a basis $|\Psi\rangle$ that are the eigenstates of $\hat{H}$ defined by:

$$
\hat{H}=\left(\hat{p}-g T^{a} A^{a}\right)^{2}+\frac{g}{2} \sigma_{\mu \nu} T^{a} F^{a \mu \nu} .
$$

which is a part of the one loop effective action $S$ of Equation (5).

As an application to this idea, we first consider the case of a constant electric field in the $z$ direction (direction of the beam in the heavy ion collision). In this case, we choose a gauge such that we can take $A_{z}^{a}=E^{a} t$. Thus the second part of Equation (6) can be written in this form

$$
\frac{g}{2} \sigma_{\mu \nu} T^{a} F^{a \mu \nu}=\mathrm{i} g E^{a} T^{a} \sigma_{3} \otimes\left(\begin{array}{ll}
0 & 1 \\
1 & 0
\end{array}\right)
$$

The Hamiltonian becomes

$$
\hat{H}=\hat{p}_{t}^{2}-\hat{p}_{x}^{2}-\hat{p}_{y}^{2}-\left(\hat{p}_{z}-g T^{a} E^{a} t\right)^{2}+\frac{g}{2} \sigma_{\mu \nu} T^{a} F^{a \mu v} .
$$

After a straightforward algebra one can find the following eigenvalues of the Hamiltonian $\hat{H}$

$$
E_{n}^{p_{x}, p_{y}, p_{z}, \Lambda_{i}, \lambda_{j}}=-\hat{p}_{T}^{2}-g \lambda_{j}(2 n+1)+\mathrm{i} g \Lambda_{i} \lambda_{j} .
$$

where $\Lambda_{i}$ are the eigenvalues over the Dirac matrices such that $\Lambda_{1}=\Lambda_{3}=1$, and $\Lambda_{2}=\Lambda_{4}=-1$. And $\lambda_{j}$, with $j=1,2,3$, are the eigenvalue for $\lambda=T^{a} E^{a}$ over the group space and are given by [18].

$$
\lambda_{1}=\sqrt{\frac{C_{1}}{3}} \cos \theta, \quad \lambda_{2}=\sqrt{\frac{C_{1}}{3}} \cos (2 \pi / 3-\theta), \quad \lambda_{3}=\sqrt{\frac{C_{1}}{3}} \cos (2 \pi / 3+\theta),
$$

${ }^{1}$ see Ref. [18] and reference therein. 
with $\theta$ given by

$$
0 \leq \cos ^{2}(3 \theta)=3 C_{2} / C_{1}^{3} \leq 1
$$

where

$$
C_{1}=E^{a} E^{a}, C_{2}=\left[d_{a b c} E^{a} E^{b} E^{c}\right]^{2} .
$$

Using the obtained eigenvalues of the Hamiltonian $\hat{H}$, the effective action becomes

$$
2 S=\mathrm{i} \int_{0}^{\infty} \frac{\mathrm{d} s}{s} \sum_{i=1}^{4} \sum_{j=1}^{3} \frac{1}{(2 \pi)^{3}} \int \mathrm{d}^{4} x \int \mathrm{d}^{2} p_{T} \mathrm{e}^{-\mathrm{is}\left(p_{T}^{2}+m^{2}\right)-s \epsilon}\left[\sum_{n=0}^{\infty}\left|g \lambda_{j}\right| \mathrm{e}^{s g \lambda_{j}(2 n+1)-s g \Lambda_{i} \lambda_{j}}-\frac{1}{2 s}\right] .
$$

Performing the i and $n$ summations we found

$$
2 S=\mathrm{i} \int_{0}^{\infty} \frac{\mathrm{d} s}{s} \sum_{j=1}^{3} \frac{1}{4 \pi^{3}} \int \mathrm{d}^{4} x \int \mathrm{d}^{2} p_{T} \mathrm{e}^{-\mathrm{is}\left(p_{T}^{2}+m^{2}\right)-s \epsilon}\left[\left|g \lambda_{j}\right| \frac{\cosh s g \lambda_{j}}{\sinh s\left|g \lambda_{j}\right|}-\frac{1}{s}\right] .
$$

which is the same results as Ref. [18]. Clearly, the one loop magnetic effective action can be found upon the following substitution $E^{a} \rightarrow-\mathrm{i} B^{a}$. Therefore

$$
2 S^{(m)}=\mathrm{i} \int_{0}^{\infty} \frac{\mathrm{d} s}{s} \sum_{j=1}^{3} \frac{1}{4 \pi^{3}} \int \mathrm{d}^{4} x \int \mathrm{d}^{2} p_{T} \mathrm{e}^{-\mathrm{is}\left(p_{T}^{2}+m^{2}\right)-s \epsilon}\left[\left|g \lambda_{j}\right| \frac{\cos s g \lambda_{j}}{\sin s\left|g \lambda_{j}\right|}-\frac{1}{s}\right] .
$$

\section{Pair Production in Non-Perturbative QCD}

Now, in the same manner as in Ref. [18] we may derive the non-perturbative quarks (antiquarks) production per unit time, per unit volume and per unit transverse momentum from a given constant chromo-electric field $E^{a}$. Thus as done in Ref. [18] we can find that

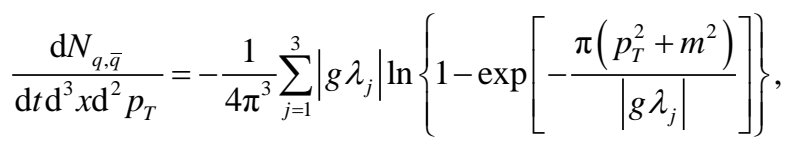

where $m$ is the effective mass of the quark and the eigenvalues $\lambda_{j}$ are given above.

\section{Application to Heavy Ion Collisions}

Let's consider the situation of two relativistic heavy nuclei colliding and leaving behind a semi-classical gluon field which then non-perturbatively produces gluon and quark-antiquark pairs via the Schwinger mechanism [19]. As estimated in Ref. [24] for Au-Au collision at RHIC collider with $R \approx 10 \mathrm{fm}$ and center-of-mass energy $\approx 200 \mathrm{GeV}$ per nucleon, the initial energy density is $\rho \approx 100 \mathrm{GeV}^{4}$ and $C_{1} \sim 100 \mathrm{GeV}^{4}$. For our analysis we take $\theta=0$ which can be justified by the sensitivity check that has been made in Ref. [24] where it has been found that the production rate is not very sensitive to $C_{2}$.

In Figure 1 we plot the rate of quark production as a function of the transverse momentum for two values of $\alpha_{s}=0.3$ (used in [25]) and $\alpha_{s}=0.4$ with initial energy density $\rho \approx 100 \mathrm{GeV}^{4}$. Clearly seen from this figure that the production rate decrease with $p_{T}$ and becomes negligible at $p_{T} \sim 3 \mathrm{GeV}$. The obtained $p_{T}$ distribution for quark (antiquark) production can be used to fix the initial conditions for the QGP in heavy ion collision at the RHIC and the LHC colliders.

\section{Conclusion}

In this note we have proposed a method for calculating the vacuum to vacuum transition amplitude in the presence of the non-abelian background field. The method can be applied to a general background field and it can be updated to study the non-perturbative soft gluon production [26]. Also, we have evaluated the rate for 


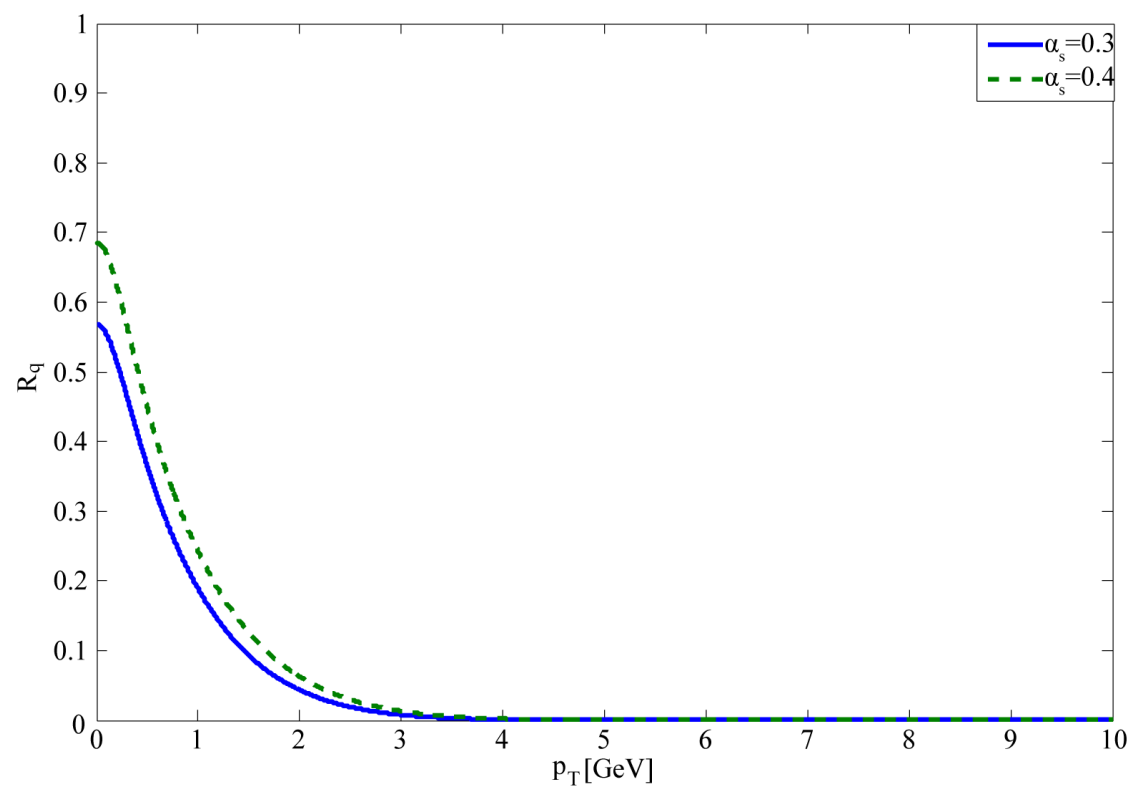

Figure 1. (Color online) Transverse production rate for quarks for $C_{1}=100 \mathrm{GeV}^{4}$ for $\alpha_{\mathrm{s}}=0.3,0.4$, as a function of $p_{T}$. For simplicity we denote here the quark production rate given in Equation (16) by $R_{q}$. We take $\theta=0, m=m_{q} \approx 1 / 3 \mathrm{GeV}$.

quark (antiquark) production in a constant chromo-electric field $E^{a}$. These results are used to determine the quark (antiquark) production rate in heavy ion collision.

\section{References}

[1] Collins, J.C. and Perry, M.J. (1975) Physical Review Letters, 34, 1353. http://dx.doi.org/10.1103/PhysRevLett.34.1353

[2] Karsch, F., Laermann, E. and Peikert, A. (2000) Physics Letters B, 478, 447-455. http://dx.doi.org/10.1016/S0370-2693(00)00292-6

[3] Hamieh, S., Letessier, J. and Rafelski, J. (2003) Physical Review A, 67, Article ID: 014301. http://dx.doi.org/10.1103/PhysRevA.67.014301

[4] Hamieh, S., Redlich, K. and Tounsi, A. (2004) Journal of Physics G, 30, 481, http://dx.doi.org/10.1088/0954-3899/30/4/007

[5] Baym, G. (1984) Physics Letters B, 138, 18-22. http://dx.doi.org/10.1016/0370-2693(84)91863-X

[6] Kajantie, K. and Matsui, T. (1985) Physics Letters B, 164, 373-378. http://dx.doi.org/10.1016/0370-2693(85)90343-0

[7] Eskola, K.J. and Gyulassy, M. (1993) Physical Review C, 47, 2329. http://dx.doi.org/10.1103/PhysRevC.47.2329

[8] Nayak, G.C. and Ravishankar, V. (1997) Physical Review D, 55, 6877. http://dx.doi.org/10.1103/PhysRevD.55.6877

[9] Nayak, G.C. and Ravishankar, V. (1998) Physical Review C, 58, 356. http://dx.doi.org/10.1103/PhysRevC.58.356

[10] Bhalerao, R.S. and Nayak, G.C. (2000) Physical Review C, 61, Article ID: 054907. http://dx.doi.org/10.1103/PhysRevC.61.054907

[11] Kluger, Y., Eisenberg, J.M., Svetitsky, B., Cooper, F. and Mottola, E. (1991) Physical Review Letters, 67, 2427; http://dx.doi.org/10.1103/PhysRevLett.67.2427

[12] Kluger, Y., Eisenberg, J.M., Svetitsky, B., Cooper, F. and Mottola, E. (1992) Physical Review D, 45, 4659. http://dx.doi.org/10.1103/PhysRevD.45.4659

[13] Kharzeev, D. and Tuchin, K. hep-ph/0501234.

[14] McLerran, L. and Venugopalan, R. (1994) Physical Review D, 50, 2225. http://dx.doi.org/10.1103/PhysRevD.50.2225

[15] Dietrich, D.D., Nayak, G.C. and Greiner, W. (2001) Physical Review D, 64, Article ID: 074006. http://dx.doi.org/10.1103/PhysRevD.64.074006

[16] Gyulassy, M. and McLerran, L. (1997) Physical Review C, 56, 2219. http://dx.doi.org/10.1103/PhysRevC.56.2219 
[17] Dietrich, D.D. Physical Review D, 7.

[18] Nayak, G.C. and Van Nieuwenhuizen, P. (2005) Physical Review D, 72, 125010. http://dx.doi.org/10.1103/PhysRevD.72.125010

[19] Schwinger, J. (1951) Physical Review, 82, 664. http://dx.doi.org/10.1103/PhysRev.82.664

[20] Claudson, M., Yildiz, A. and Cox, P.H. (1980) Physical Review D, 22, 2022. http://dx.doi.org/10.1103/PhysRevD.22.2022

[21] Casher, A., Neuberger, H. and Nussinov, S. (1979) Physical Review D, 20, 179. http://dx.doi.org/10.1103/PhysRevD.20.179

[22] Glendenning, N.K. and Matsui, T. (1983) Physical Review D, 28, 2890. http://dx.doi.org/10.1103/PhysRevD.28.2890

[23] Dunne, G.V. (2004) Heisenberg-Euler Effective Lagrangians: Basics and Extensions, hep-th/0406216. In: Shifman, M., et al., Eds., From Fields to Strings, Vol. 1, 445-522.

[24] Cooper, F., Dawson, J.F. and Mihaila, B. (2008) Physical Review D, 78, 117901. http://dx.doi.org/10.1103/PhysRevD.78.117901

[25] Aurenche, P. and Zakharov, B.G. arXiv:1205.6462 [hep-ph].

[26] work in progress. 